\title{
Short-term memory: Effects of auditory and visual similarity'
}

\author{
RICHARD S. CIMBALO AHD KENNETH R. LAUGHERY \\ STATE UNIVERSITY OF NEW YORK AT BUFFALO
}

The effects of five variables on short-term memory for letters were studied: Auditory Similarity, Visual Similarity, Presentation Rate, Sequence Length, and Blocks of Trials. Performance varied directly with Presentation Rate and inversely with Sequence Length and Auditory Similarity. Performance improved over blocks with high auditory and high visual similarity, and decreased under the low conditions of these variables. The improvement is attributed to leaming to ignore redundant cues and attend to dues that permit discrimination. The decrement is attributed to fatigue and/or waning attention.

The literature on short-term memory (STM) reveals three types of stimulus similarities that affect performance. Laughery (1963), Sperling (1963), Conrad \& Hull (1964), and Wickelgren (1965) have demonstrated that STM is superior for acoustically distinct letters as opposed to acoustically similar letters. Semantic similarity has been shown by Baddeley (1966) to degrade performance in STM. Hintzman (1965) has argued that kinesthetic similarity also has deleterious effects.

While Sperling (1963) and Rudov (1966) have explored the role of visual information storage and retrieval for a task involving very-short-term memory (VSTM), no evidence exists to demonstrate the role of visual similarity in STM. Baddeley attempted to study this effect with words but reported difficulty in separating visual from acoustic similarity. It is felt that letters may be more manageable units to determine effects of visual similarity; hence, the purpose of the present study is to investigate, using letters, the role of visual similarity in STM relative to the effects of acoustic similarity.

Method

One hundred and twenty students from an introductory psychology course participated as Ss. The design was a 2 by 2 by 3 by 2 by 2 factorial. Auditory Similarity, high vs. low, was manipulated as a between$S$ variable and consisted of letters that were aurally similar or distinct respectively. Visual Similarity, high vs. low, also a between-s variable, consisted of visually similar or distinct items respectively. Presentation Rate was a between-S variable and included three rates: 30,60 , and 120 items $/ \mathrm{min}$. Sequence Length was manipulated as a within-s variable and included sequences with seven or nine letters. Finally, Blocks of Trials was analyzed by comparing the first 10 trials to Trials 11-20.

Four groups of four consonants were selected on the basis of phonemic and visual similarity. The four groups were: High Visual-High Auditory (BPDG); Low Visual-High Auditory (BGTV); High Visual-Low Auditory (KXVM); and Low Visual-Low Auditory (TYRQ). Visual Simllarity was based upon common characteristics (straight line, curves, and position of these elements) and was found to conform very well to Gibson's (1963) more elaborate patterns of distinctive graphemes. Gibson distinguished each grapheme as to whether or not it possessed each of 12 graphic features. The high visual sets in this experiment had 29 distinctive features (determined by summing the features shared between each of the letters in a stimulus set), whereas the low visual sets had a total of 20 distinctive features. Gibson also presented a confusion matrix obtained in a recognition task with pre-school children. There were 25 confusions between the letters in the high visual similarity groups and seven between the letters in the low visual similarity groups.

Twenty lists, 10 each of lengths 7 and 9, were constructed using each of the four symbol sets. The letters were assigned at random with the restriction that each letter occur at least once in each list. The 20 lists were randomly assigned to two blocks of 10 lists with the restriction that five lists of each length occur in each of the two blocks.

The sequences were recorded at the three different rates using a Roberts model No. 1057 tape recorder. The sequences were then presented to Ss who responded by writing the letters on answer sheets, which contained the correct number of positions, seven or nine, for each of the 20 trials. Ss were instructed to record the letters in the correct serial position and were encouraged to guess.

Resulis

The mean number of items correct per sequence was used as the performance measure. In order to be correct an item had to be recorded in the proper position. An analysis of variance showed the main effects of three variables to be significant. Auditory Similarity was significant, $F=24.26, d f=1 / 108, p<$ .001 , with means of 5.1 and 4.5 for low and high respectively. Sequence Length, $F=511.22$, df $=1 / 108$, $\mathrm{p}<.001$, had means of 5.6 and 4.0 for lengths 7 and 9 . Finally, Presentation Rate, $F=15.62$, df $=2 / 108, p<$ .001 , showed means of $5.3,4.5$, and 4.6 for the 30,60 , and $120 \mathrm{items} / \mathrm{min}$. rates respectively.

The Blocks by Presentation Rate interaction was significant, $F=4.10, d f=2 / 108, p<.05$. The data showed an Improvement over Blocks for the 30 and 60 items/ 


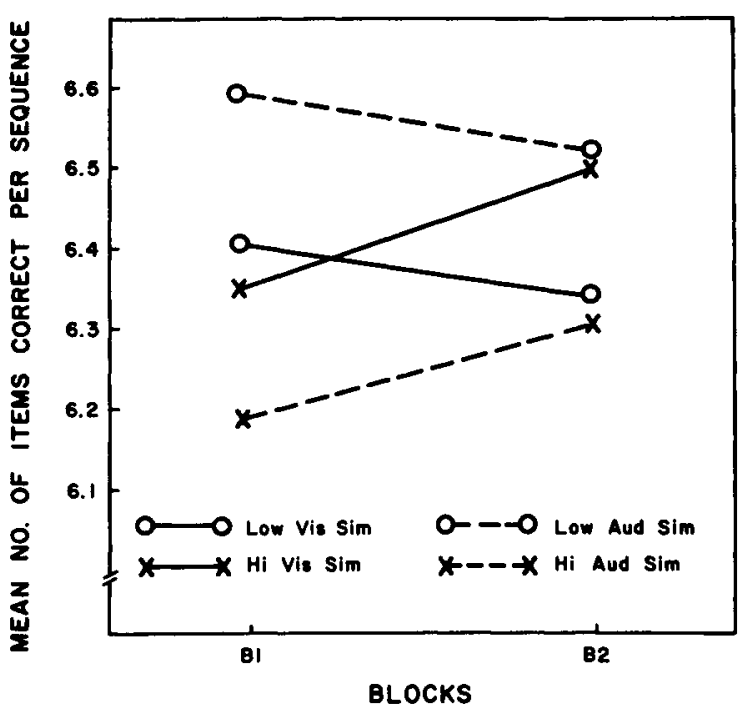

Fig. 1. Mean number of items correct per sequence for visual and auditory similarity.

min. conditions and a slight decrement for the 120 items/min. condition.

The Blocks by Sequence Length interaction was significant, $F=17.02$, df $=1 / 108, p<.001$, with an improvement in performance for length 9 from Block 1 to Block 2 and a decrement for length 7 .

The Blocks by Visual Similarity interaction was also significant, $F=7.64$, $\mathrm{df}=1 / 108, p<.001$. Performance improved with high visual similarity, whereas there was a slight decrement with low visual similarity.

Blocks also interacted significantly with Auditory Similarity, $F=8.27, \mathrm{df}=1 / 108, \mathrm{p}<.001$. Performance improved with high auditory similarity, whereas there was a slight decrement for low auditory similarity. The data underlying this interaction as well as the Blocks by Visual Similarity interaction are plotted in Fig. 1.

\section{Discussion}

The main effects of Sequence Length, Presentation Rate, and Auditory Similarity are consistent with previous findings in STM.

The various interactions with Blocks indicate changes in performance with practice. The Blocks by Presentation Rate interaction seems to indicate that if $\mathrm{S}$ has sufficient time between stimuli a more optimal strategy for processing the Information will evolve. For example, strategies involving rehearsal and/or chunking may be utilized at the slower rates. The faster rates, however, may preclude efficient use of these strategies, and in fact a decrement occurs, perhaps due to fatigue or waning attention.

The interactions between Blocks and sequence Length, Auditory Similarity and Visual Similarity indicate similar effects. Performance under the more difficult levels of these variables improves with practice, and a decrement occurs with the less difficult conditions. This finding seems consistent with typical learning data, wherein greater task difficulty results in a lesser slope (more errors) in the learning curve. With less task difficulty, an optimum performance may be achieved relatively early.

Although Visual Similarity does not appear to be a major factor in STM, the interaction with Blocks indicates that it does play a role in storage and retrieval. The improvement with high visual similarity and high auditory similarity might result from the fact that Ss learn to ignore the redundant information (the similar cues) and more effectively process those cues that enable discrimination. The decrements that occur with low visual and low auditory similarity can probably be attributed to fatigue or loss of interest.

\section{References}

Baddeley, A. D. Short-term memory for word sequences as a function of acoustic, semantic and formal similarity. Quart. J. exp. Psychol, 1966, 18, 362-365.

Conrad, R., \& Hull, A. J. Information, acoustic confusion and memory span. Brit. J. Psychol., 1964, 55, 429-432.

Gibson, E., Osser, H., Shiff, W., \& Smith, J. A basic research program on reading. Cooperative Research Project No. 639 (Final Report), Cornell University, Ithaca, N. Y, 1963.

Hintzman, D. L. Classification and aural coding in short-term memory. Psychon. Sci., 1965, 3, 161-162.

Langhery, K. R. Effects of symbol set on immediate memory. Amer. Psychologist, 1963, 18, 415 (Abstract).

Sperling, G. A. A model for visual memory tasks. Hum. Factors, $1963,5,19-31$

Rudov, M. H. Dimensionality in human information storage. J. exp. Psychol., 1966, 71, 273-281.

Wickelgren, W. A. Short-term memory for phonemically similar lists. Amer. J. Psychol., 1965, 78, 567-574.

\section{Hote}

1. This research was supported by Public Health Service Grant MH-11595. 